\title{
Role of the grating profile in Smith-Purcell radiation at high energies
}

\author{
J. H. Brownell \\ Department of Physics and Astronomy, Dartmouth College, Hanover, New Hampshire 03755-3528, USA \\ G. Doucas* \\ Sub-Department of Particle Physics, University of Oxford, Denys Wilkinson Building, Oxford OX1 3RH, United Kingdom
}

(Received 6 May 2005; published 2 September 2005)

\begin{abstract}
The passage of a finely focused electron beam near the surface of a periodic metallic grating produces radiation known as Smith-Purcell radiation. This paper presents an analysis of the role of the grating profile in the case of echelle-type gratings whose period consists of two facets only. Particular emphasis is placed on the ultrarelativistic regime and a comparison is made with recent experimental results in this region. It is shown that the details of the profile of the grating play an important role in the optimization of the radiated energy. The behavior of higher order modes and the limitations of the surface current description of the radiative process are also discussed briefly.
\end{abstract}

DOI: 10.1103/PhysRevSTAB.8.091301

PACS numbers: $41.60 .-\mathrm{m}, 41.75 . \mathrm{Ht}, 41.85 . \mathrm{Ew}$

\section{INTRODUCTION}

Fifty years after Smith and Purcell first reported the emission of visible radiation arising out of the interaction of an electron beam with a periodic structure [1], the process that now bears their name is being considered for a number of diverse applications. The most promising of these are its use as a compact, tunable source of farinfrared radiation and as a diagnostic tool for the determination of the longitudinal (time) profile of intense, picosecond long bunches of charged particle beams. Whatever the potential application, a good theoretical understanding of the emission process is essential, so that sensible extrapolations can be made, especially in the highly relativistic regime. The various attempts to do this can be broadly classified under two categories: in the first, the emission is described as the diffraction of the electromagnetic field of the electron [2-5], while in the second the emission is ascribed to the acceleration of charges induced on the grating surface by the passage of the electron beam [1,6,7]. Although we use the term "electron," SmithPurcell (SP) radiation is produced by any charged particle. This paper concentrates on the second approach, which has been supported by experimental evidence well into the relativistic regime $[8,9]$. However, recent experiments [10] at higher energies $(855 \mathrm{MeV})$ have cast doubt on the validity of this treatment and, indeed, on the efficiency of the SP process at very high energies.

Whichever theoretical description is chosen, one important parameter that appears in the formulas for the angular distribution of the radiated energy is the "grating efficiency" or "radiation factor," usually symbolized by most authors by $R^{2}$. The so-called strip grating where the period is assumed to consist of a section of metal, oriented parallel to the beam direction, followed by empty space has

\footnotetext{
*Corresponding author.
}

been discussed in some detail [6,7]. It has the advantage of an exact analytical solution for the evaluation of $R^{2}$, but it does not represent a physically realistic device. Solid blazed gratings present almost the opposite situation: the simple analytical solution is no longer possible but they do represent practical devices that can be used, as will be shown later, to extract significant power from the electron beam. The solution of the equation for the angular distribution of radiated energy, in this case, can be achieved by numerical methods. However, it would be useful to derive some simple formulas that can be used in order to select the optimum grating profile for a particular experimental situation. The availability of such formulas would obviate the need for repeated applications of the full computer calculation with randomly selected grating parameters, until one converges on a design that appears to be optimum. The objective of this paper is to examine the behavior of $R^{2}$ for blazed gratings within the terms of the surface current treatment and to predict its behavior at high values of the relativistic factor $\gamma$. It also considers some potentially interesting properties of radiation emitted at orders higher than the fundamental.

It should be stated at the outset that this is a multiparameter problem, depending amongst other things on the intended application for the SP radiation. It is, therefore, impossible to give answers to all scenarios. Since one potential use of SP radiation is as a diagnostic tool for highly relativistic beams, we concentrate on the role of the grating profile in that regime. The discussion is restricted to the case of the incoherent emission from a dilute, highly relativistic beam. This is a useful starting point for the analysis of SP radiation, but it excludes a significant number of working accelerators where the bunch is short enough for coherence to be dominant. Coherence effects, which are only mentioned briefly in this paper, enter as an additional factor to the emitted power [6]. 


\section{SURFACE CURRENTS}

The coordinate system used in this paper is the standard spherical polar one, with the beam propagating along the $z$ axis and with the $x$ axis perpendicular to the plane of the grating (see Fig. 1). It is assumed that observation of the emitted radiation is taking place far away from the grating, at an angle $\theta$ relative to the beam axis. In order to keep the number of formulas to a minimum, we omit the details of the mathematical analysis and note only some of the "milestones" in the treatment of the various expressions. The details of the physical model have been presented elsewhere [6,7] and we take as starting point the formula for the energy $d I$ emitted in solid angle $d \Omega$, arising from the interaction of a single electron with a grating of period $\ell$ and total length $Z$ :

$$
\left(\frac{d I}{d \Omega}\right)_{1}=2 \pi q^{2} \frac{Z}{\ell^{2}} \frac{n^{2} \beta^{3}}{(1-\beta \cos \theta)^{3}} R^{2} \exp \left[-\frac{2 x_{0}}{\lambda_{e}}\right] .
$$

Other quantities appearing in (1) are the charge $q$, the order of the emitted radiation $(n)$, the height of the electron trajectory above the grating surface $\left(x_{0}\right)$, the relativistic factors $\beta=v / c$ and $\gamma=\left(1-\beta^{2}\right)^{-1 / 2}$, and the "evanescent wavelength" $\lambda_{e}$ defined by
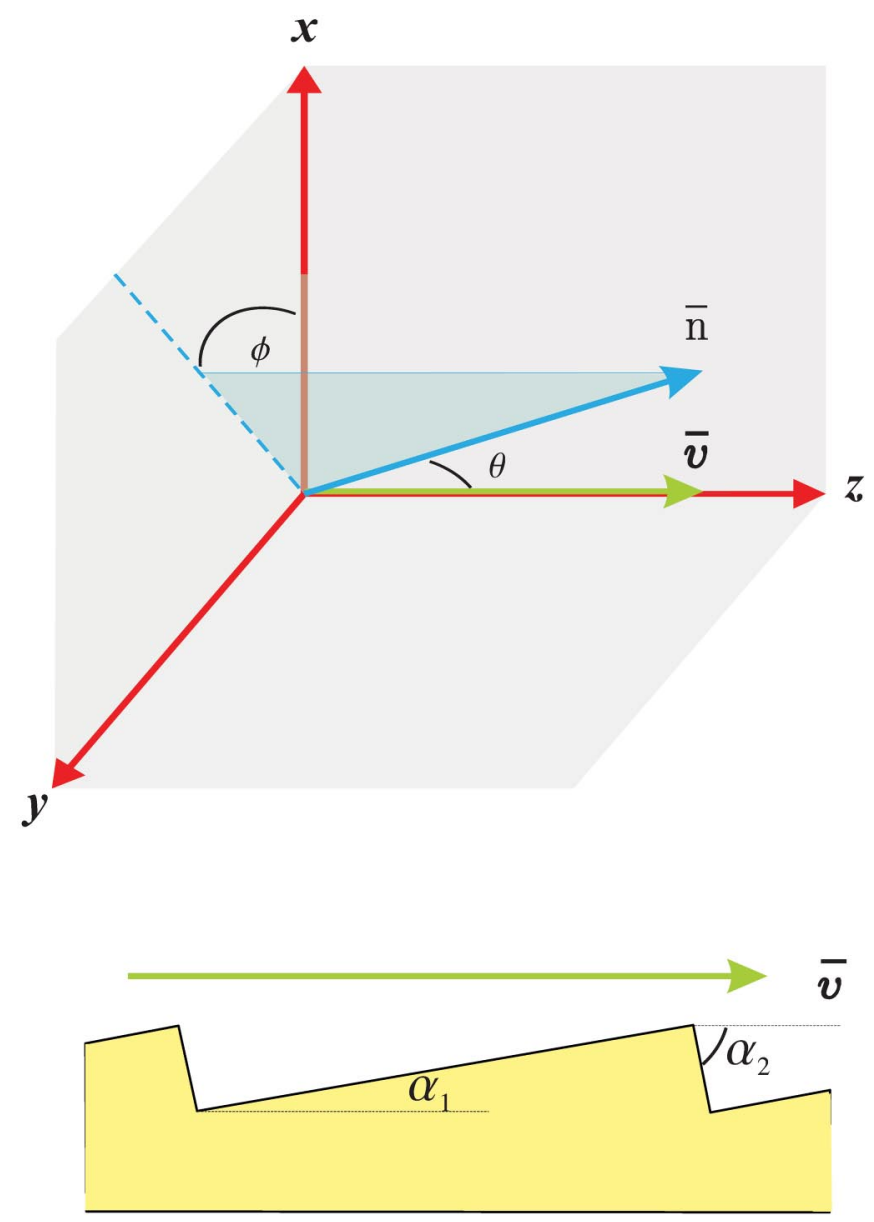

FIG. 1. (Color) Schematic of coordinate system and of grating.

$$
\lambda_{e}=\frac{\lambda}{2 \pi} \frac{\beta \gamma}{\sqrt{1+\beta^{2} \gamma^{2} \sin ^{2} \theta \sin ^{2} \phi}},
$$

where $\phi$ is the azimuthal angle of emission. The exponential dependence agrees with the intuitive notion that the low wavelength cutoff in the SP spectrum is limited by the size of the image charge footprint, $x_{0} / \gamma$, for relativistic electrons. In the surface current treatment of the emission process, the symbol $R^{2}$ represents the field contribution from each tooth and is defined by the expression

$$
R^{2}=|\bar{n} \times(\bar{n} \times \bar{G})|^{2} .
$$

The vector $\bar{G}$ that appears in (2) is complex and is discussed in Sec. II B. However, it can be noted here that it is preferable to resolve $\bar{G}$ into two mutually perpendicular directions, both of them perpendicular to the emission direction $\bar{n}$, and with unit vectors $\bar{\varepsilon}_{||}$and $\bar{\varepsilon}_{\perp}$, respectively. The vector $\bar{\varepsilon}_{||}$lies in the plane of vectors $\bar{n}, \bar{z}$. For observation that is sufficiently far from the grating, the line of sight to each tooth is practically parallel, and

$$
\begin{aligned}
\bar{\varepsilon}_{\| \mid} & =(\cos \theta \cos \phi, \cos \theta \sin \phi,-\sin \theta), \\
\bar{\varepsilon}_{\perp} & =(-\sin \phi, \cos \phi, 0) .
\end{aligned}
$$

Therefore, $R^{2}$ can be resolved into two polarization components $R_{\|}^{2}$ and $R_{\perp}^{2}$, where

$$
R_{\|}^{2}=\left|\bar{\varepsilon}_{\|} \cdot \bar{n} \times(\bar{n} \times \bar{G})\right|^{2}=\left|\bar{\varepsilon}_{\|} \cdot \bar{G}\right|^{2},
$$

and similarly for $R_{\perp}^{2}$.

In order to calculate the radiated energy from a real beam it is necessary to know the distribution of the particles above and across the grating ( $x$ and $y$ directions, respectively), as well as the longitudinal (time) one. The energy is then given by

$$
\left(\frac{d I}{d \Omega}\right)_{N_{e}}=\left(\frac{d I}{d \Omega}\right)_{1}\left(N_{e} S_{\mathrm{inc}}+N_{e}^{2} S_{\mathrm{coh}}\right) .
$$

For uncorrelated distributions, $\quad \rho(x, y, t=z / v)=$ $X(x) Y(y) T(t)$, the "coherent integral" $S_{\text {coh }}$ in the above expression is given by

$$
\begin{aligned}
S_{\mathrm{coh}}= & \left|\int_{0}^{\infty} X e^{-\left(x-x_{0}\right) / \lambda_{e}} d x\right|^{2}\left|\int_{-\infty}^{\infty} Y e^{-i k_{y} y} d y\right|^{2} \\
& \times\left|\int_{-\infty}^{\infty} T e^{-i \omega t} d t\right|^{2},
\end{aligned}
$$

while the "incoherent integral" $S_{\text {inc }}$ is given by

$$
S_{\mathrm{inc}}=\int_{0}^{\infty} X e^{-2\left(x-x_{0}\right) / \lambda_{e}} d x,
$$

where it is convenient to identify $x_{0}$ as the beam center.

For bunched beams whose bunch length is short enough to be comparable to or shorter than the wavelength of the radiation, the second term inside the parenthesis of (3) will dominate and there is "coherent enhancement" of the emitted radiation (by a factor approaching $N_{e}$ ). This will 
be critically dependent on the last integral in $S_{\text {coh }}$, which is the Fourier transform of the time profile of the bunch.

If the bunch length is long compared to the wavelength, the shape of the bunch becomes irrelevant because $S_{\text {coh }}$ is negligible and the situation resembles that of a continuous, dilute beam. In this case, the contributions of $N_{e}$ electrons will add together in an incoherent way and the radiated energy will be just $N_{e} S_{\text {inc }}$ times that of a single electron:

$$
\left(\frac{d I}{d \Omega}\right)_{N_{e}} \cong\left(\frac{d I}{d \Omega}\right)_{1}\left(N_{e} S_{\mathrm{inc}}\right) .
$$

The present paper deals with incoherent emission. For such a beam it is probably more convenient to use an expression for the radiated power $P$ (rather than intensity) in terms of the beam current $J$ :

$$
\frac{d P}{d \Omega}=2 \pi q J \frac{Z}{\ell^{2}} \frac{n^{2} \beta^{3}}{(1-\beta \cos \theta)^{3}} e^{-2 x_{0} / \lambda_{e}} R^{2} S_{\text {inc }} .
$$

A full discussion can be found in Refs. [6,8].

In Ref. [7], the peak power was derived for the simple case of a zero blaze strip grating. Here we analyze the factors appearing in Eq. (3a) separately in order to optimize arbitrary grating profiles. Generally, the grating efficiency can be optimized independently of the parameters governing the electron-grating coupling strength. In effect, a grating can be "blazed" for a particular wavelength and order, even for nonechelle profiles. Before discussing in detail the term $R^{2}$, we consider first the other factors that appear in Eq. (3a).

\section{A. Form factor $f$}

Using the Smith-Purcell relationship for the emitted wavelength of diffraction order $n$,

$$
\lambda=\frac{\ell}{n}(1 / \beta-\cos \theta),
$$

the emitted power can be written

$$
\frac{d P}{d \Omega}=f R^{2} S_{\text {inc }}
$$

if the "form factor," a measure of the coupling efficiency between beam and grating, is defined as

$$
f=2 \pi q J \frac{Z \ell}{n \lambda^{3}} \exp \left[-\frac{4 \pi x_{0}}{\gamma \beta \lambda} \sqrt{1+(\gamma \beta \sin \theta \sin \phi)^{2}}\right] .
$$

There are several general comments on the form of $f$ worth noting: (a) The azimuthal dependence of the evanescent length constrains Smith-Purcell radiation around the normal plane $(\phi=0)$. (b) The evanescent coupling length is proportional to $\gamma \beta$ and so can be quite long at high energy. (c) For a fixed wavelength, operating on the first order radiates more power. (d) The exponent suggests that the beam center should be zero. While this is correct for a single electron, a realistic beam has a finite minimum waist radius $\left(r_{0}\right)$. More of the electrons are scraped by the grating as the height decreases, as indicated by a drop in $S_{\text {inc }}$. For a
Gaussian profile, the optimum height is $x_{0}=r_{0}$ (rms). (e) A perfectly collimated beam would accommodate a grating of any length. A realistic beam has a finite range of collimation ("characteristic length," "Rayleigh range," or depth of focus) $L_{\text {coll }}=\left(r_{0}^{2} \beta \gamma\right) / \varepsilon_{N}$, where $\varepsilon_{N}$ is the normalized emittance. Beam scraping at both ends constrains the optimum length to $Z=L_{\text {coll }}$, given the waist is at the center of the grating.

Typically, we aim to maximize radiated power at a particular wavelength $\left(\lambda_{\max }\right)$. Incorporating the constraints of comments (d) and (e) above and assuming that the beam focus (i.e., $r_{0}$ ) is adjustable, differentiation with respect to $r_{0}$ shows that the function has a maximum $f_{\max }$ at a wavelength $\lambda_{\max }$ that corresponds to an observation angle $\theta_{\max }$. These are given by

$$
\begin{aligned}
r_{0} & =\lambda_{e}^{\max } \\
\cos \theta_{\max } & =\left(\frac{1}{\beta}-\frac{n \lambda_{\max }}{\ell}\right), \\
f_{\max } & =\frac{e^{-2}}{2 \pi} \frac{q J \gamma^{3} \beta^{3}}{\varepsilon_{N}} \frac{\ell}{n \lambda_{\max }},
\end{aligned}
$$

and inspire further comments: (f) Eq. (4a) implies that the beam waist should be adjusted to match the evanescent length for the wavelength of interest. If this is not feasible, then aim as many electrons within an evanescent length of the grating surface as possible. (g) Eq. (4b) is the SmithPurcell relation reversed. It is clear that if the first order wavelength is much less than the period, then the emission angle is approximately constant. This occurs for highly relativistic electrons $(\gamma \gg 1)$ when emission peaks in the forward direction. In this case, all diffraction orders, up to the limit mentioned above, will appear in the same direction.

\section{B. Grating factor $\boldsymbol{R}^{\mathbf{2}}$}

When the grating profile over one period is comprised of $N$ intersecting planar facets, the vector $\bar{G}$ in (2) is the vector sum of the contribution from each facet: $\bar{G}=\sum_{j=1}^{N} \bar{G}_{j}$. The contribution of the $j$ th facet is given by

$$
\begin{aligned}
\bar{G}_{j}= & \left(\tan \alpha_{j}, 2 i k_{y} \lambda_{e} \tan \alpha_{j}, 1\right) \exp \left[\left(\frac{1}{\lambda_{e}}-i k_{x}\right) x_{j 1}\right. \\
& \left.+i\left(\frac{k}{\beta}-k_{z}\right) z_{j 1}\right] \frac{\exp \left[i D_{j}\left(z_{j 2}-z_{j 1}\right)\right]-1}{i D_{j} \ell},
\end{aligned}
$$

with

$$
D_{j}=\frac{k}{\beta}-k_{z}-k_{x} \tan \alpha_{j}-i \frac{\tan \alpha_{j}}{\lambda_{e}},
$$

where $\alpha_{j}$ is the blaze angle and $\left(x_{j 1}, z_{j 1}\right),\left(x_{j 2}, z_{j 2}\right)$ are coordinates of the leading and trailing facet edges referenced to the beginning of the period. Many of the features of high energy SP radiation can be gleaned from the blazed strip grating example. In this case, the tooth profile consists 
of only one thin but perfectly reflective facet. For emission in the normal plane $(\phi=0)$, the radiation is polarized in the $(n, z)$ plane; i.e., $R_{\perp}^{2}=0$ and the grating efficiency then reduces to

$$
\begin{aligned}
R^{2} & =R_{\|}^{2} \\
& =2 \frac{\beta^{2}}{k^{2} \ell^{2}} \frac{\sin ^{2}[\theta-\alpha]}{(1-\beta \cos \theta)(1-\beta \cos [\theta-2 \alpha])} e^{\left(x_{1}+x_{2}\right) / \lambda_{e}}\left(\cosh \left[\frac{\tan \alpha}{\lambda_{e}}\left(z_{2}-z_{1}\right)\right]-\cos \left[\left(\frac{1}{\beta}-\frac{\cos (\theta-\alpha)}{\cos \alpha}\right) k\left(z_{2}-z_{1}\right)\right]\right) .
\end{aligned}
$$

The emission pattern is characterized by maxima in the forward direction and at the specular reflection angle $(\theta=$ $2 \alpha$ ), due to the denominator, with a null in the facet plane $(\theta=\alpha)$. Secondary maxima appear with angular distance from these major peaks. Emission outside of the plane of incidence is symmetric about $\phi=0$, with a maximum at $\phi=0$ for $|\theta-\alpha|>\alpha$ but a local minimum for $|\theta-\alpha|<$ $\alpha$, and increasing perpendicular polarization component for increasing $\phi$.

The similarity of this emission pattern to transition radiation (TR) is expected. Transition radiation is the case when the facet extends to the electron position $\left(x_{0}\right)$, joined to another extending above, to form a seamless infinite plane. Then the contribution from the upper plane is out of phase with that of the lower, producing emission cones with axial null at $\theta=0$ and $\theta=2 \alpha(\phi=0)$. SP radiation logically should be thought of as the interference pattern from a periodic array of diffracting elements, the advantages of SP radiation being magnification by the number of array elements and angular dispersion. Because SP radiation is a simple generalization of the TR configuration, the SP radiation intensity should obey all of the confirmed scaling laws of the latter.

\section{Echelle gratings}

As noted above, at least two facets are required to form a solid grating. In general, $R^{2}$ is a complicated expression involving the emission angle, the relativistic factor $\gamma$ and geometrical details of the grating profile. The algebra involved in its evaluation is straightforward but tedious and the only practical approach is a numerical evaluation. Nevertheless, considerable simplification is possible for the special case where the emitted radiation is observed at an azimuthal angle $\phi=0\left(R_{\perp}^{2}=0\right)$ and the grating is of the "echelle" type; i.e., it consists of two facets per period, the first having an angle $\alpha_{1}$ relative to the beam and the second vertical to the beam axis $\left(\alpha_{2}=-90^{\circ}\right)$. Assuming further that $\gamma \gg 1$, the expression for $R^{2}$ can be rewritten:

$$
\begin{aligned}
R^{2}= & R_{\|}^{2} \\
\cong & {\left[\frac{1}{\sin \theta-\tan \alpha_{1}(1+\cos \theta)}\right]^{2} } \\
& \times \frac{2 e^{-k h / \beta \gamma}[\cosh (k h / \beta \gamma)-\cos (k h \sin \theta)]}{k^{2} l^{2}},
\end{aligned}
$$

where $h=l \tan \alpha_{1}$ is the height of the indentation of the grating profile. In spite of its apparent difference, this expression is very similar to that derived in the previous section for a single, inclined facet: the first fraction in the above expression has a maximum at $\theta=2 \alpha_{1}$, and the second fraction can easily be shown to be equal to the square bracket of (5). Thus, the blaze angle $\alpha_{1}$ should be set equal to half the value of $\theta_{m}$, as defined by (4); this maximizes both $f$ and the first fraction of (6) at the same emission angle, irrespective of the emission order. This is the origin of the "bunching" of all the emission orders, especially for high values of $\gamma$, in the case of blazed gratings.

The rather complicated form of the second fraction in (6) does not allow for an easy differentiation with respect to $\theta$ and the determination of the maximum. Neither can it be assumed that the quantities

$$
\frac{k h}{\beta \gamma} \equiv \frac{2 \pi n h}{\gamma l(1-\beta \cos \theta)} \quad \text { and } \quad k h \sin \theta,
$$

which appear in the fraction, are necessarily small, especially for values of $\theta$ approaching zero. However, for emission angles a few degrees away from zero and for $\gamma \gg$ 1 it is possible to have $k h / \beta \gamma \ll 1$. In this case, and remembering that $h=l \tan \alpha_{1}$, the second fraction in (6) tends to

$$
\begin{aligned}
& \frac{2 e^{-k h / \beta \gamma}[\cosh (k h / \beta \gamma)-\cos (k h \sin \theta)]}{k^{2} l^{2}} \Rightarrow \frac{4 \sin ^{2}\left(\frac{k h \sin \theta}{2}\right)}{(k l)^{2}} \\
& =\tan ^{2} \alpha_{1} \sin ^{2} \theta \frac{\sin ^{2}\left[\frac{k h \sin \theta}{2}\right]}{\left[\frac{k h \sin \theta}{2}\right]^{2}} .
\end{aligned}
$$

This becomes zero at $\theta=0^{\circ}$ and $\theta=180^{\circ}$, and has a broad maximum, of order $\tan ^{2} \alpha_{1}$, in the region of $\theta=90^{\circ}$. Superimposed on that are oscillations due to the last term, which is of the form $\sin ^{2} x / x^{2}$. As far as its minima are concerned, it is easy to show that for a given blaze angle $\alpha_{1}$, they occur when $\tan \frac{\theta}{2}=\frac{n}{p} \tan \alpha_{1}$ given $\beta \cong 1$. The integer $p$ refers to the first, second, etc., minimum of the expression $\sin ^{2} x / x^{2}$. Thus, for first order emission $(n=1)$, the first "dip" $(p=1)$ will be at $\theta \cong 2 \alpha_{1}=\theta_{m}$ and the next one at $\theta \cong \alpha_{1}$. On the other hand, for fifth order emission, the first minimum will be at an angle substantially higher than $\alpha_{1}$ and the other minima will be "piling up" around the blaze angle.

In summary, one can say that, for high values of $\gamma$, the expression for $R^{2}$ tends to 


$$
R^{2} \Rightarrow\left[\frac{1}{\sin \theta-\tan \alpha_{1}(1+\cos \theta)}\right]^{2} \tan ^{2} \alpha_{1} \sin ^{2} \theta \frac{\sin ^{2}\left[\frac{k h \sin \theta}{2}\right]}{\left[\frac{k h \sin \theta}{2}\right]^{2}} .
$$

This is essentially independent of $\gamma$ but its behavior as a function of $\theta$ is not monotonic, exhibiting local maxima and minima especially for orders higher than 1 .

\section{SELECTION OF GRATING PARAMETERS}

The preceding information can be exploited in the design of the grating for a specific experiment. We emphasize again that it is not possible to give a universal answer to all situations. Depending on what exactly the user wants to achieve and on the particular experimental conditions, different approaches are possible.

We consider here the problem of designing the appropriate echelle grating to radiate the maximum energy at a particular wavelength $\lambda_{\max }$ given an electron beam of known energy and normalized beam emittance. Assume the beam can be focused to satisfy Eq. (4a). The grating period affects both the emission angle and form factor. As extreme forward detection tends to be difficult, a compromise must be made between a practicable emission angle $\theta_{\max }$ and greater power. The blaze angle $\alpha_{1}$ of the grating should be set equal to $\theta_{\max } / 2$. If the presence of multiple coincident orders is problematic, then increasing the blaze angle while reducing the period pushes the emission to higher $\theta$ and angularly disperses the different orders into distinct peaks with slightly diminished power. Although the above approach is strictly valid for a particular type of echelle grating and for beams of high $\gamma$ and low emittance, it will nevertheless provide a reasonable initial approximation in cases where one or more of these conditions are not satisfied.

As a specific example, we consider the case of a hypothetical experiment with a grating placed near a $\gamma=1000$ beam. If the desired peak wavelength were $\lambda_{\max }=$ $10 \mu \mathrm{m}$, then (4a) implies the waist need only be $x_{0}=$ $1.6 \mathrm{~mm}$. Furthermore, if it is desired to collect this radiation at an emission angle of, say, $\theta \cong 10^{\circ}$, then (4b) gives a period $l=0.658 \mathrm{~mm}$ for first order. The blaze angle must then be set to $\alpha_{1}=5^{\circ}$. These are practicable values for a grating. The evaluation of $R^{2}$ requires numerical methods. This has been done for emission orders 1 and 2 . The results of the calculation are shown in Fig. 2(a). Note the peak of the grating factor at an emission angle equal to twice the blaze angle of the grating. This is more obvious in the calculation for $n=2$. The total emitted power in orders 1 , 2, and 3 is shown in Fig. 2(b). The calculation is based on Eq. (3). All three emission orders have a peak at $\theta \cong 10^{\circ}$, as anticipated by our optimization criteria, although orders 2 and 3 exhibit additional maxima at other emission angles, due to the influence of $R^{2}$.

However, it is not always possible or advisable to make the wavelength of interest coincide with the maximum of the form factor. For example, if the wavelength of interest were $\lambda=100 \mu \mathrm{m}$, then the maximum of the form factor would be at $x_{0}=16 \mathrm{~mm}$ and the grating length would be impractically long. The longest reasonable grating length would then govern the optimum design instead. The choice of the angle of emission is, to a great extent, up to the user. If this is set at $\theta=20^{\circ}$, then this would define a period of $1.66 \mathrm{~mm}$ and a blaze angle of $\alpha_{1}=10^{\circ}$. Again, these are practicable values. Figures 3(a) and 3(b) show $R^{2}$ and the emitted power, respectively. For this longer period grating, the three orders still peak at approximately the same emission angle, around the expected value of $20^{\circ}$. Figure 4 is a more vivid demonstration of the role of the radiation factor $R^{2}$. The grating parameters are the same as those of Fig. 3, but the blaze is "reversed" with a consequent loss of efficiency by a factor of about 25 .

It is interesting to note that dependence of the grating efficiency on energy is weak and, as expected from (7), $R^{2}$ becomes essentially independent of $\gamma$ above $\gamma>100$, approximately. This is shown in Fig. 5, which plots $R^{2}$ for a wide range of energies. The grating used is that of Fig. 3.

\section{DISCUSSION AND COMPARISON WITH EXPERIMENTS}

As mentioned earlier, the surface current analysis of the SP radiation has worked well for low energies $(\gamma=10)$ and preliminary results indicate that it predicts correctly at $50 \mathrm{MeV}$. However, in a recent experiment at higher energy $(\gamma=1674)$, the observed output was much smaller than anticipated [10]. It is, therefore, of some interest to apply the predictions of our analysis to the gratings used in that experiment. Details of the experimental conditions can be found in the cited reference, but we highlight some of the essential features. Apart from the high energy, this was an experiment aimed at observing SP radiation in the visible part of the spectrum $(\lambda=360$ and $546 \mathrm{~nm})$. The gratings were made of glass, with a $700 \mathrm{~nm}$ coating of aluminum. Their periods were very small $(0.833 \mu \mathrm{m}$ for gratings A and B and $9.09 \mu \mathrm{m}$ for grating C) and the coupling between beam and grating was also very close $(\sim 0.1 \mathrm{~mm})$.

Figure 6 shows the calculated grating factors $R^{2}$ for these gratings. The rapid variation of $R^{2}$ with angle is obvious, especially for grating $\mathrm{B}$, which had a blaze angle of about $41^{\circ}$. Note also the significantly lower efficiency of grating $\mathrm{C}$, with a low blaze angle of $0.8^{\circ}$. The radiated energy (or power) can be calculated from (3) or (3a). Since the bunch length was about $10 \mathrm{~ns}$, which is orders of magnitude longer than the observed wavelengths, the radiation is entirely incoherent. In addition, beam intensity and radiated energy were low and, therefore, one can probably ignore any interaction between the two. The calculated radiated energies for these gratings are shown in Fig. 7(a) for A and B and in Fig. 7(b) for grating C. These plots show the energy per bunch per sr and per $\mathrm{cm}$ 

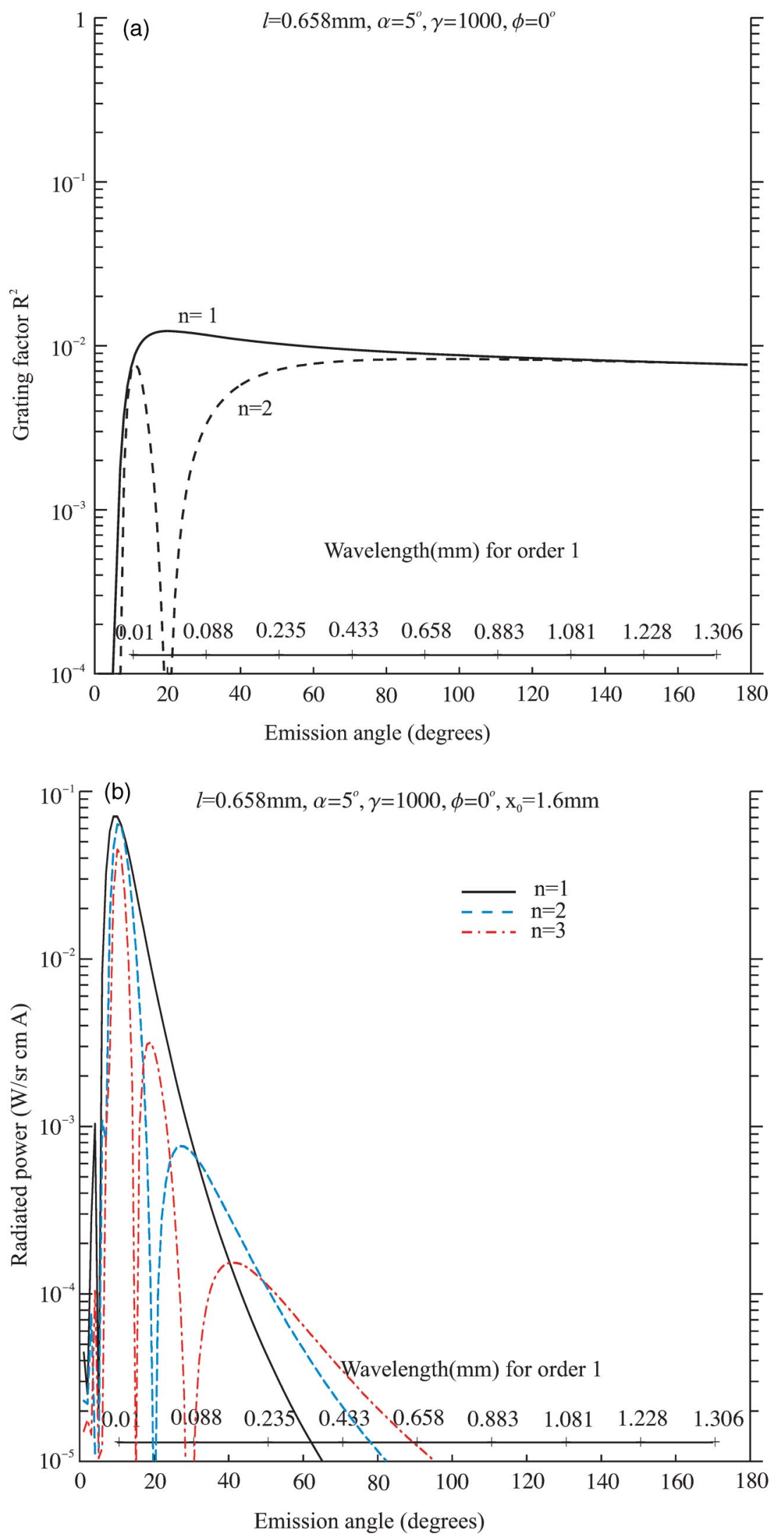

FIG. 2. (Color) (a) The grating factor $R^{2}$ (orders 1 and 2) for a grating designed for $\lambda=10 \mu \mathrm{m}$ at $\theta=10^{\circ}$. The period is $0.658 \mathrm{~mm}$ and the blaze angle $5^{\circ} ; \gamma=1000$. (b) Total differential power (W/sr cm A), in orders $1-3$, for the grating of (a). 

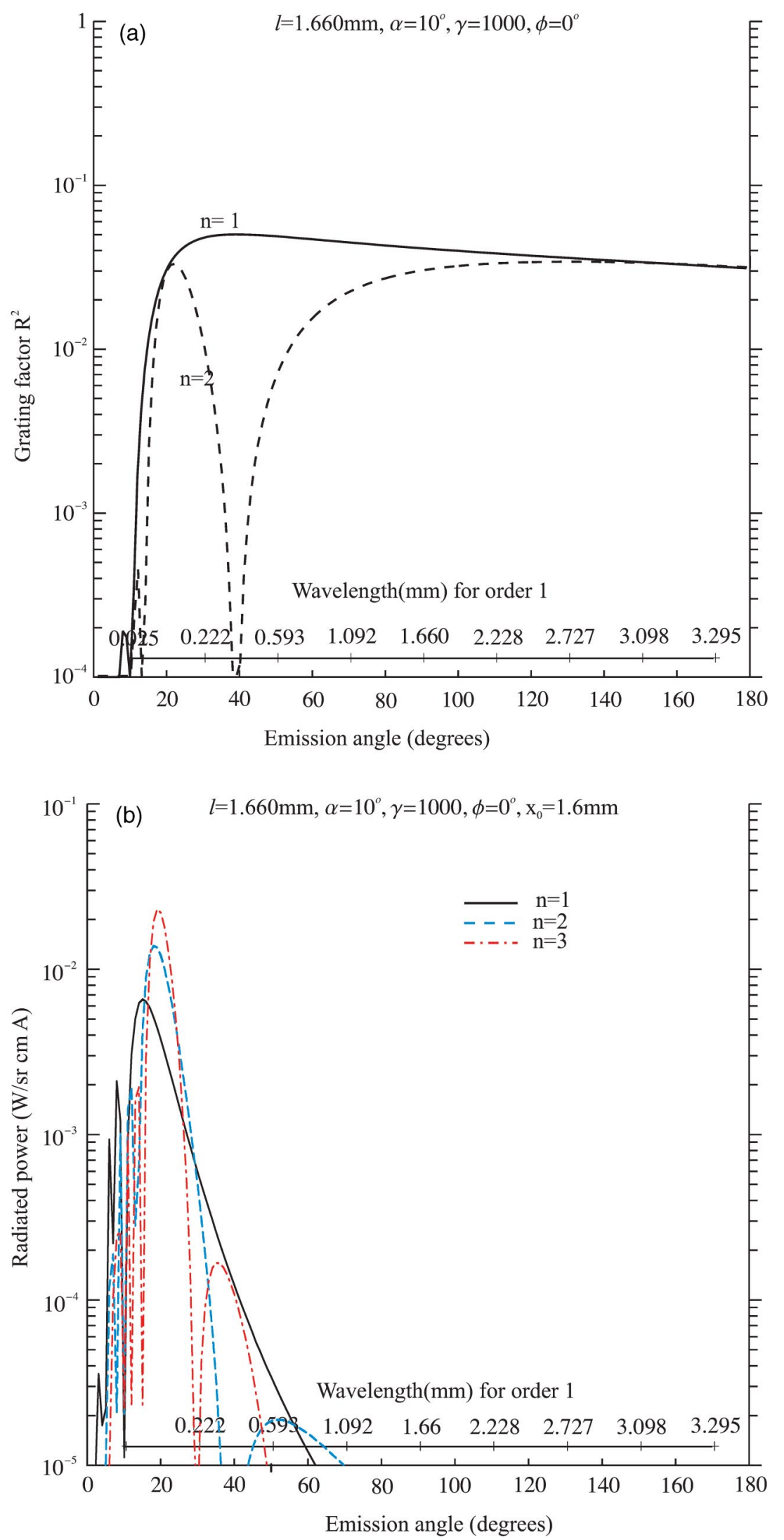

FIG. 3. (Color) (a) The grating factor $R^{2}$ (orders 1 and 2) for a grating designed for $\lambda=100 \mu \mathrm{m}$ at $\theta=20^{\circ}$. The period is $1.66 \mathrm{~mm}$ and the blaze angle $10^{\circ} ; \gamma=1000$. (b) Total differential power (W/sr cm A), in orders $1-3$, for the grating of (a). 


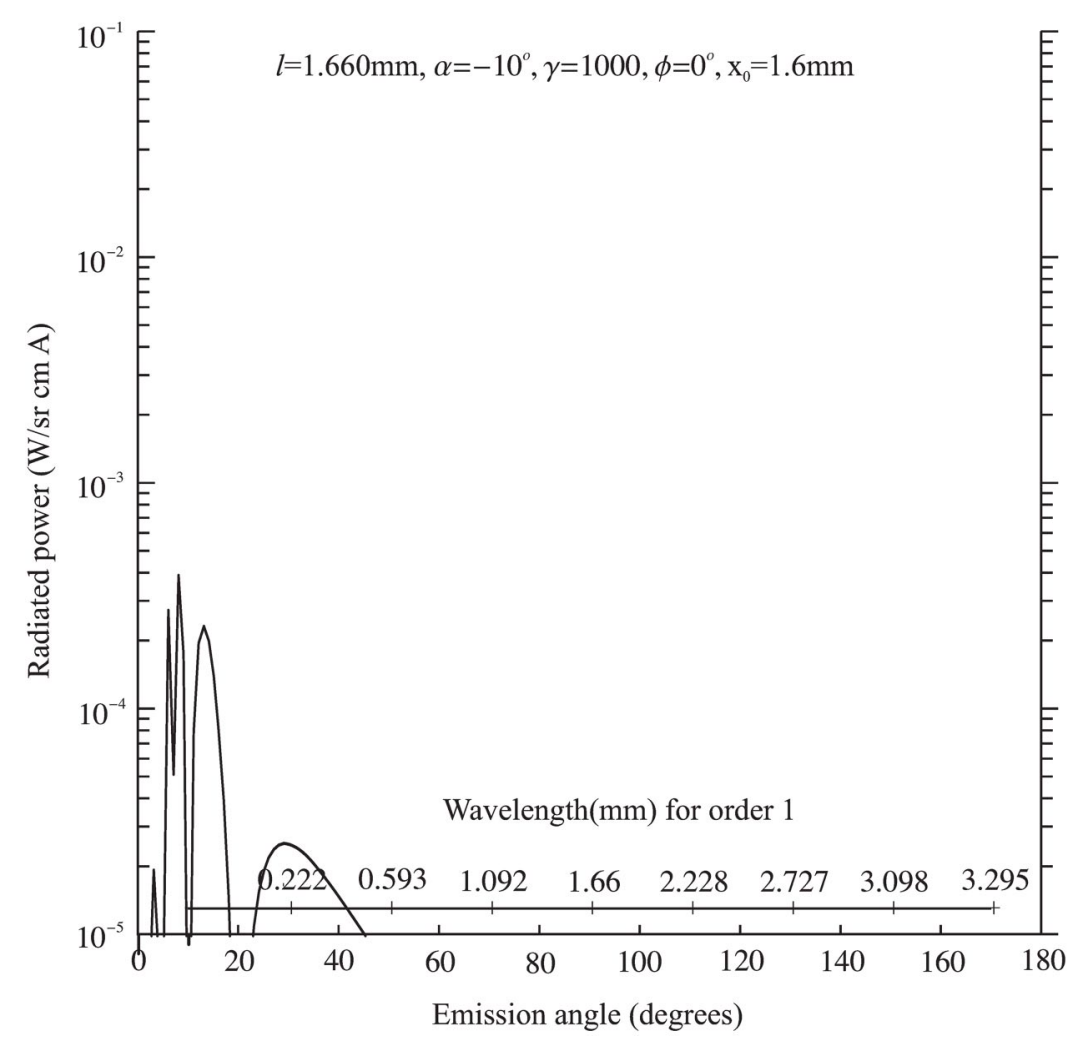

FIG. 4. The grating factor $R^{2}$ for the same grating as Fig. 3(a), but with "reverse" blazing.

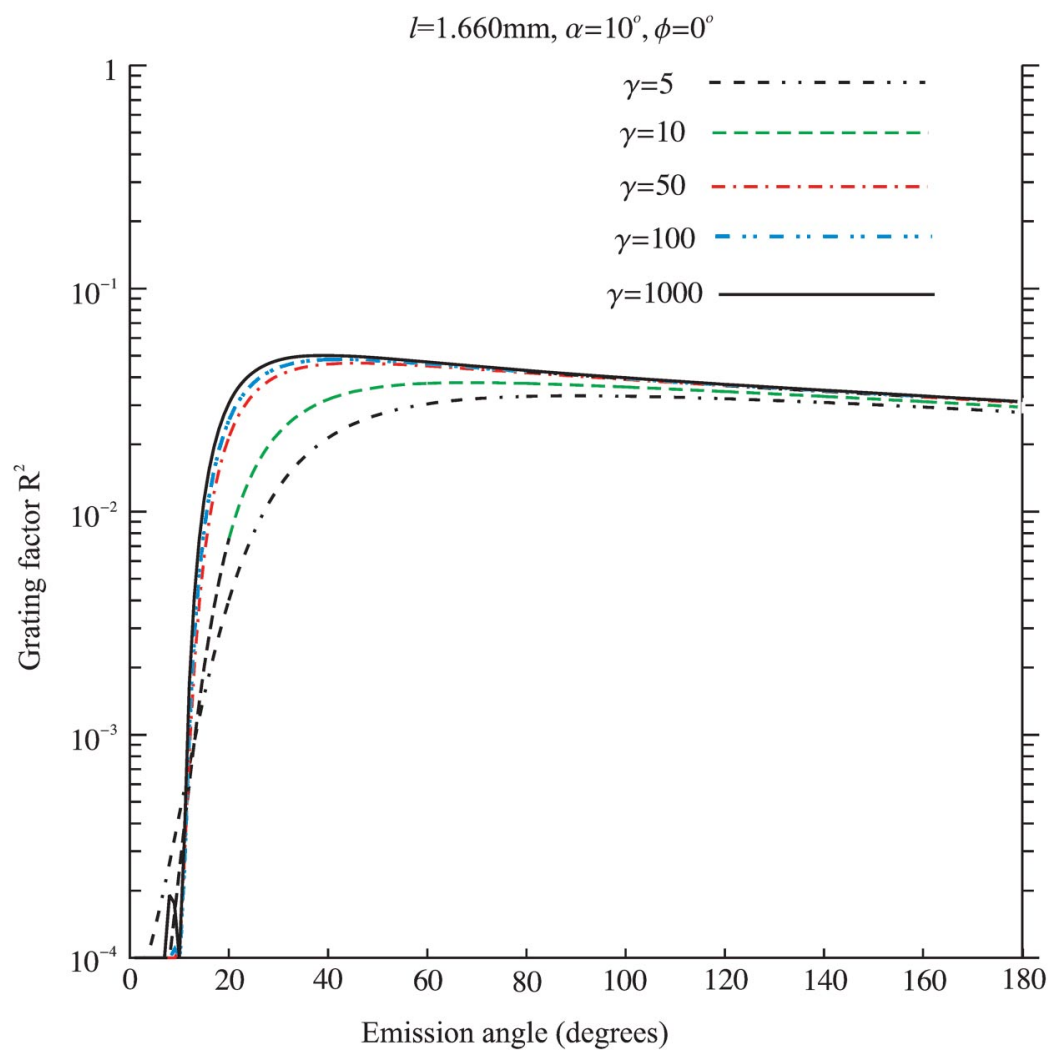

FIG. 5. (Color) Variation of $R^{2}$ with energy. Grating parameters are those of Fig. 3(a). 


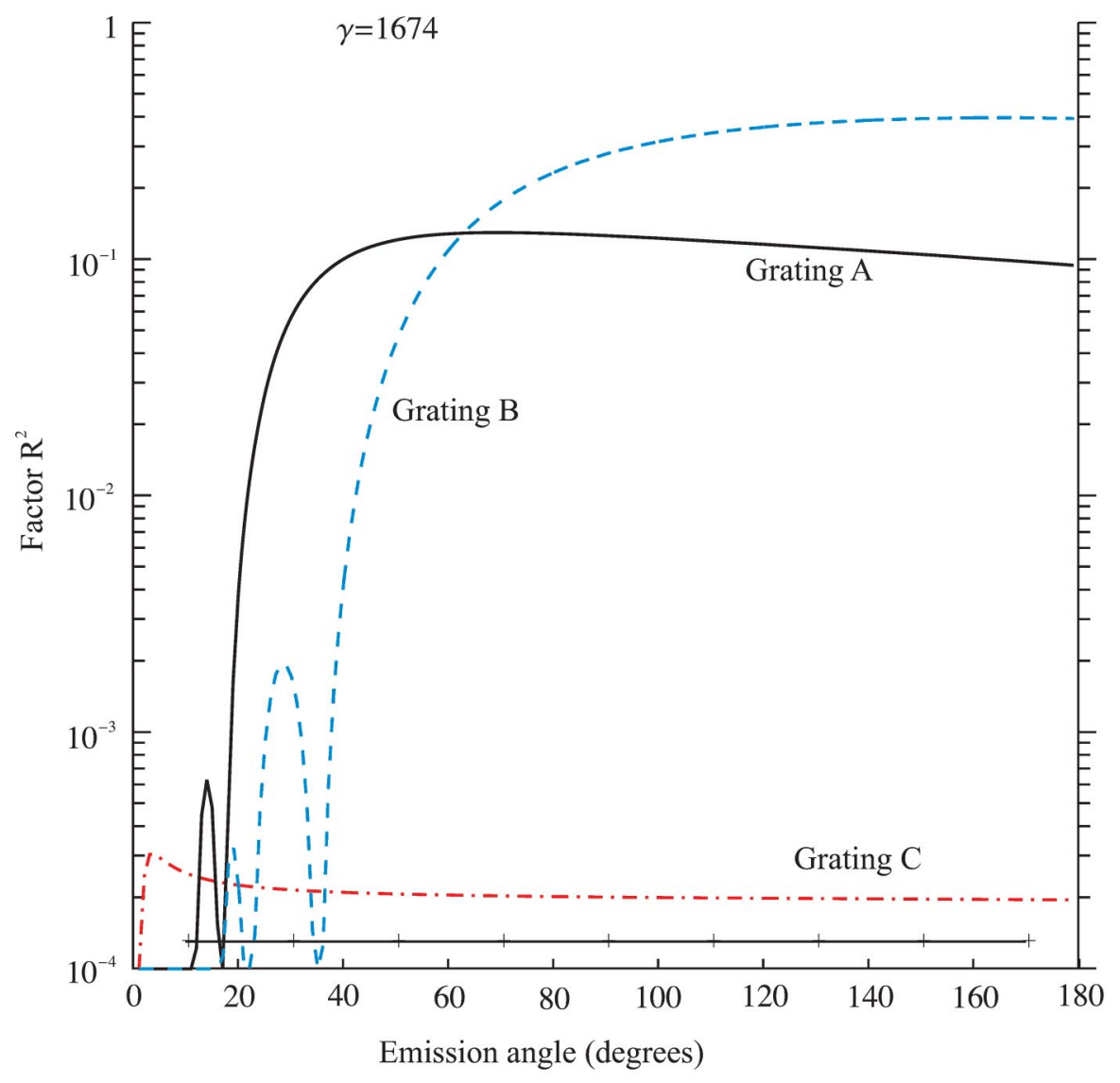

FIG. 6. (Color) The grating factor $R^{2}$ for gratings A, B, and C of Ref. [10].

length of grating. We have used Eq. (3a), assuming a Gaussian longitudinal profile, with $90 \%$ of the particles inside $10 \mathrm{~ns}$. The same result, expressed as radiated power, can be obtained from Eq. (3). The lower efficiency of $\mathrm{C}$ is reflected in the different scale used in Fig. 7(b). These calculations have assumed a Gaussian bunch, with $90 \%$ of the particles inside $10 \mathrm{~ns}$. Also shown in 7(a) are the two emission angles that correspond to the measured wavelengths of 360 and $546 \mathrm{~nm}$ (about $55^{\circ}$ and $70^{\circ}$, respectively).

Using the value of $9 \times 10^{-12} \mathrm{~J} /(\mathrm{srcm})$ for grating $\mathrm{B}$ at $55^{\circ}$, the given number of bunches per s $(\sim 5000)$, the grating length $(2 \mathrm{~cm})$, and the detection efficiency $(\sim$ $2 \%$ ), this differential energy can be translated into about $3.3 \times 10^{9}$ photons/(s sr). According to the authors, the collected solid angle is about $52 \mathrm{msr}$, which should produce a count rate of $1.7 \times 10^{8}$ photons/s. This is about 6 orders of magnitude higher than the measured one.

In our opinion, the reasons for this large discrepancy should be sought neither in the behavior of the grating factor nor in radiative process at very high energies but in the circumstances of this particular experiment and in the basic assumption of the surface current model of SP radiation. Numerical evaluation of the grating factor indicates that it increases slowly with energy up to about $100 \mathrm{MeV}$ and, as shown in Sec. II C, it becomes essentially independent of $\gamma$ for further increases in energy. In addition, there is no physical reason to believe that the SP radiative process, which is one of a number of similar processes, would weaken with increasing beam energy. On the other hand, all the analysis presented here is based on the assumption of perfect conductivity for the grating material. This will certainly be true as long as the SP frequencies are low compared to the plasma frequency of the metal [11], which is typically between $10^{15}-10^{16}$. In the Mainz experiment the frequency was of the order of $10^{15}$, roughly similar to the plasma frequency, and it is possible that perfect conductivity cannot be assumed. It is worth noting that previous work in this wavelength region [12] but at low energy $(130 \mathrm{keV})$ has some interesting comments on the role of surface conditions, finite conductivity, etc.

Another consideration is the finite thickness of the aluminum coating of the grating. The authors speculate that their reduced signal could be explained by the existence of mirror charges $100 \mathrm{~nm}$ below the surface cancelling the surface charge effect. Their gratings were glass substrates coated with a $700 \mathrm{~nm}$ aluminum layer, which could conceivably have developed a mirror image on the underside of the coating. Using solid metal gratings would avoid this issue. 

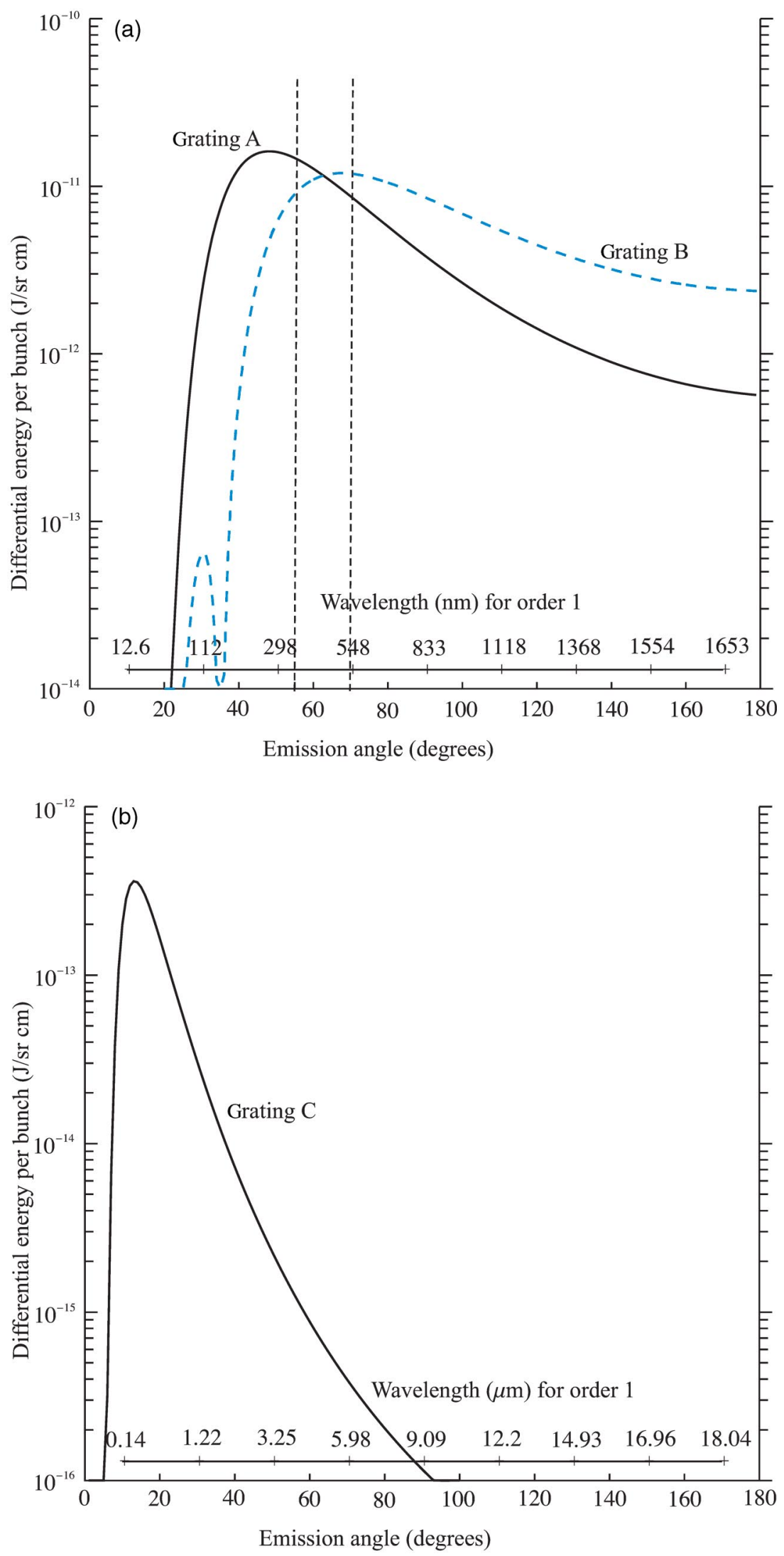

FIG. 7. (Color) (a) Calculated differential energy ( $\mathrm{J} / \mathrm{srcm}$ ) for gratings A and B of Ref. [10] (see text for details). (b) Ditto for grating $\mathrm{C}$. 


\section{SUMMARY AND CONCLUSIONS}

We have analyzed the role of the grating profile in Smith-Purcell radiation, in the highly relativistic regime. The analysis was based on the "surface current" description of the radiation process and on echelle gratings with two facets per period. Coherence effects that dominate in the case of short bunches have not been considered in this analysis. The conclusions can be summarized as follows: (i) Smith-Purcell radiation should be seen as a generalization of transition and diffraction radiation and should obey all the confirmed energy scaling laws of the latter. (ii) The grating efficiency or radiation factor $R^{2}$ is weakly dependent on energy, increasing only slightly with energy up to about $\gamma=100$ and remaining essentially unchanged thereafter. This conclusion is different from that reached in Ref. [10]. (iii) The grating efficiency factor is dependant on the emission angle and on the order of the emitted radiation. We have shown that the blaze angle $\alpha$ of the grating is important and there is an emission maximum at $\theta \cong 2 \alpha$. (iv) For highly relativistic beams there is a tendency for the first few emission orders to peak together at $\theta \cong 2 \alpha$. (v) Recent experiments at $855 \mathrm{MeV}$ have measured radiated power levels much smaller than those predicted by the surface current model. This can probably be attributed to the fact that the required frequencies in these experiments were close to the plasma frequency of the metal and, hence, the assumption of perfect conductivity of the grating was not valid, or the thin metal surface layer developed an image on the underside, which cancelled the SP radiation field. (vi) However, there is no reason to doubt that ultrarelativistic beams will produce long wavelength SP radiation at power levels that agree with existing theories. This has already been demonstrated by the work of Ishi et al. [13] and Shibata et al. [14] who have carried out experiments at 42 and $150 \mathrm{MeV}$ and have confirmed that the observed (coherent) radiation in the far infrared was in agreement with their theoretical predictions. Further experimental evidence in this regime is clearly required. (vii) In our opinion, any future applications of SP radiation will have to concentrate on the long wavelength region (far infrared) where it does have a role to play, both as a tunable source of radiation and, in the coherent regime, as a diagnostic tool for the determination of the longitudinal profile of picosecond or subpicosecond bunches.

\section{ACKNOWLEDGMENTS}

The financial support of the Particle Physics and Astronomy Research Council in the U.K. and the NSF (Grant No. ECS-0070491) in the USA is gratefully acknowledged.

[1] S. J. Smith and E.M. Purcell, Phys. Rev. 92, 1069 (1953).

[2] G. Toraldo di Francia, Nuovo Cimento 16, 61 (1960).

[3] P. M. van den Berg, J. Opt. Soc. Am. 63, 689 (1973).

[4] P. M. van den Berg, J. Opt. Soc. Am. 63, 1588 (1973).

[5] O. Haeberlé, P. Rullhusen, J. M. Salomé, and N. Maene, Phys. Rev. E 49, 3340 (1994).

[6] J.H. Brownell, J.E. Walsh, and G. Doucas, Phys. Rev. E 57, 1075 (1998).

[7] S.R. Trotz, J.H. Brownell, J.E. Walsh, and G. Doucas, Phys. Rev. E 61, 7057 (2000).

[8] G. Doucas, M. F. Kimmitt, A. Doria, G.P. Gallerano, E. Giovenale, G. Messina, H.L. Andrews, and J.H. Brownell, Phys. Rev. ST Accel. Beams 5, 072802 (2002).

[9] G. Doucas, M. F. Kimmitt, A. Doria, G. P. Gallerano, E. Giovenale, G. Messina, H.L. Andrews, and J.H. Brownell, in Proceedings of the 8th European Particle Accelerator Conference, Paris, 2002 (CERN and EPSIGA, Geneva, 2002), p. 1870.

[10] G. Kube, H. Backe, H. Euteneuer, A. Grendel, F. Hagenbuck, H. Hartmann, K. H. Kaiser, W. Lauth, H. Schöpe, G. Wagner, Th. Walcher, and M. Kretzschmar, Phys. Rev. E 65, 056501 (2002).

[11] C. Kittel, Introduction to Solid State Physics (John Wiley \& Sons, New York, 1966), 3rd ed., Chap. 8.

[12] J. P. Bachheimer, Phys. Rev. B 6, 2985 (1972).

[13] K. Ishi, Y. Shibata, T. Takahashi, S. Hasebe, M. Ikezawa, K. Takami, T. Matsuyama, K. Kobayashi, and Y. Fujita, Phys. Rev. E 51, R5212 (1995).

[14] Y. Shibata, S. Hasebe, K. Ishi, S. Ono, M. Ikezawa, T. Nakazato, M. Oyamada, S. Urasawa, T. Takahashi, T. Matsuyama, K. Kobayashi, and Y. Fujita, Phys. Rev. E 57, 1061 (1998). 